\title{
Les établissements, les hommes, le travail, l'espace
}

\section{Christian Chevandier}

\section{OpenEdition}

\section{Journals}

Édition électronique

URL : https://journals.openedition.org/rhcf/1716

DOI : 10.4000/rhcf.1716

Éditeur

Rails \& histoire

Édition imprimée

Date de publication : 1 décembre 2003

Pagination : 11-20

ISBN : 0996-9403

ISSN : 0996-9403

\section{Référence électronique}

Christian Chevandier, «Les établissements, les hommes, le travail, l'espace », Revue d'histoire des chemins de fer [En ligne], 28-29 | 2003, mis en ligne le 12 décembre 2014, consulté le 22 avril 2022 URL : http://journals.openedition.org/rhcf/1716 ; DOI : https://doi.org/10.4000/rhcf.1716 


\section{Christian CHEVANDIER}

\section{Les établissements, les hommes, le travail, I'espace}

«L'époque contemporaine, elle, s'est attaquée au site. Un chemin de fer est venu couper l'entrée de l'allée, un lotissement la borde à droite ; le canal et des ateliers fournissent, à gauche, un décor sans prestige partiellement dissimulé par un rideau d'arbres ${ }^{1}$. » C'est ainsi qu'il y a moins de vingt ans étaient présentés les Alyscamps dans le plus lu des guides touristique de Provence. L'auteur de ces lignes aurait été fort étonné d'apprendre que des historiens pourraient porter intérêt à ces sites industriels et ferroviaires, aux hommes et aux femmes, plus rares, qui y ont œuvré, et qu'une séance de colloque serait même consacrée à ces architectures « sans prestige » qui accèdent même au statut de patrimoine. L'époque engendre des problématiques, elle révèle également des fonds d'une grande qualité pour l'histoire de ces sites de travail. Outre les archives " classiques ", celles du centre d'archives historiques de la SNCF au Mans et de celui de Béziers, qui recèle, avec les dossiers professionnels des agents, les sources d'une fort prometteuse histoire quantitative, les fonds d'images nous aideront à répondre à nos questions, que ce soient les films, dont l'approche est largement balisée par la thèse de Michel Ionascu ${ }^{2}$, ou les photographies, dont l'activité du Groupe archives Quatre-Mares nous a dans un ouvrage livré un échantillon des milliers de plaques argentiques qu'il a découverts ${ }^{3}$, sans méconnaitre les très riches fonds constitués par des photographes, comme celui d'Yves Broncard. N'oublions pas, enfin, ces si précieuses « archives sensibles » auxquelles nous a initiés Noëlle Gérôme ${ }^{4}$. Archives écrites, mais sans doute également quelque peu archives sensibles, ces textes de cheminots, les autobiographies bien sûr, en privilégiant celles dont l'écriture n'a pas été sollicitée, voire guidée, mais également

1- «Provence », Guide vert, Clermont-Ferrand, Michelin, 1983, p. 62.

2- Michel Ionascu, Cheminots argentiques : l'image d'un groupe social dans le cinéma et l'audiorisuel français, thèse de recherches cinématographiques et audiovisuelles, université de Paris III, 1999 (voir Cheminots et cinéma. La représentation d'un groupe social dans le cinéma et l'audiovisuel français, Paris, L'Harmattan, 2001, 400 p.)

3- Groupe Archives Quatre-Mares, Les Ateliers de Quatre-Mares. Huit décennies au service de réparation des locomotives, Paris, Éditions La Vie du rail, 1996. Michel Groguennec, auteur d'une communication dans le présent volume, est membre du Groupe.

4- Noëlle Gérôme (sous la dir. de), Archives sensibles. Images et objets du monde industriel et ouvrier, Cachan, Éditions de l'ENS, 1995. 
d'autre textes, comme ce trésor pour une histoire des représentations cheminotes que constitue le recueil de poèmes écrits sur les Ateliers du Prado 5 .

Cette manifestation scientifique présente l'esquisse d'une histoire totale dans le cadre d'une approche précise, sur un temps long, de la monarchie de Juillet à nos jours, qui est le temps de l'histoire des chemins de fer. Cette histoire commence en France avec les frères Seguin, et dans ce domaine, la thèse capitale de Michel Cotte ${ }^{6}$ nous permet d'appréhender plus exactement son moment initial. La démarche historique nous autorise à saisir le fonctionnement du système ferroviaire, par une mise en perspective sur un temps long, par des éléments de comparaison entre les différents types d'établissements. Histoire des établissements, bien sûr, qui relève de l'histoire de l'industrie, de celle des entreprises, des rapports entre les différentes structures économiques, entre celles-ci et l'État. La dimension internationale apparait dans ce colloque, le détour se fait par l'Australie, la Belgique, l'Italie, l'Angleterre, plus largement le vieux continent, qui permet de distinguer et de préciser d'éventuelles spécificités. Par ailleurs, la capacité de débusquer des caractères fallacieusement ferroviaires est d'autant plus réelle lors de ce colloque qu'y participent de nombreux chercheurs ayant étudié ou étudiant d'autres groupes professionnels. Cette approche internationale nous permet de nous attarder sur des éléments qui nous semblent sûrs, trop sûrs. La comparaison entre le monde du rail belge et celui de l'Australie est fort stimulante, pour des espaces nationaux qui sont fort distincts. Ils diffèrent certes par leur taille, mais divergent plus radicalement sans doute par les logiques des dynamiques ferroviaires. Plus fondamentalement, ce sont leurs rôles dans la construction des espaces nationaux qui les distinguent. Si Fernand Braudel, dans une controverse avec Pierre Chaunu ${ }^{7}$, a pu expliquer au début des années 1980 que, plus que Jeanne d'Arc, c'est le chemin de fer qui a fait la France, que n'auraitil dit de la Belgique lorsque l'État sinon la nation se construit alors que se tracent les lignes du réseau ferré ? A l'étranger, bien souvent, et cela

5- Jean-Pierre Ostende, Le Pré de Buffalo Bill. Mémoire collective des cheminots des ateliers du Prado, Marseille, Via Valeriano, 1990.

6- Michel Cotte, Innovation et transfert de technologies, le cas des entreprises de Marc Seguin (France 1815-1835), thèse d'histoire des techniques, Ecole des hautes études en sciences sociales, 1995, $1042 \mathrm{p}$.

7- Fernand Braudel, Une Legon d'histoire, Paris, Arthaud-Flammarion, 1986 ; Pierre Chaunu, La France, Paris, Pluriel, 1982. 
nous permet une mise en perspective mieux encadrée, plus que celle de l'activité de transport, c'est la spécificité de l'intervention de l'État qui peut apparaître la plus prégnante.

Cette esquisse d'une histoire totale est d'abord celle des établissements, des entreprises, de ces ateliers destinés initialement à la construction, puis voués à la maintenance. Mais une construction qui s'élabore dans un premier temps autour de méthodes et de produits, de machines, d'outils qui n'ont rien de spécifiquement ferroviaire, des réparations qui se révèlent de fait être des productions, lorsque la pièce que l'on remplace est unique, doit être fabriquée sur place, avant qu'une normalisation des matériels ne s'impose de fait, accroissant considérablement la productivité et réduisant d'autant les effectifs de ces sites industriels. Histoire des dépôts également, où l'on établit que les scansions peuvent être semblables, avec cette rupture formidable qu'a constitué le passage à la traction électrique. Les travailleurs, de tous niveaux de qualification, le mécanicien et l'ouvrier d'atelier comme l'ingénieur, constituent un groupe social, des groupes sociaux qui sont appréhendés à travers leur évolution, leurs mutations. Histoire de carrières, histoire de représentations, histoire de formations aussi, car la formation de la corporation cheminote, le Making d'Edward P. Thompson ${ }^{8}$, passe par la formation du cheminot, formation longtemps sur le tas jusqu'à ce que soient organisés pendant la Grande Guerre des centres d'apprentissage, avant même la loi Astier. Pour la formation, les carrières, le recours est indispensable à l'approche longitudinale. Approche nécessaire également pour percevoir, pour apercevoir oserais-je écrire, les femmes qui travaillent dans ces lieux d'hommes. Les historiens, les historiennes surtout, nous ont permis ces deux dernières décennies de connaitre le travail des femmes ${ }^{9}$. Son appréhension passe aujourd'hui par celle du travail des hommes, tout comme la compréhension du monde presque exclusivement masculin des cheminots nécessite la perception de ce que furent et de ce que sont les cheminotes, dans les deux sens du terme, les épouses de cheminots ${ }^{10}$ mais aussi, surtout, les

8- Edward P. Thompson, The Making of the English Working Class, Harmondsworth, Penguin Books, 1972.

9- Sylvie Schweitzer, Les Femmes ont toujours travaillé. Une histoire de leurs métiers, XIX et $X X^{*}$ siècles, Paris, Odile Jacob, 2002.

10- À propos de l'expression « faire la cheminote », voir Jean-Paul Burdy, Le Soleil noir. Formation sociale et mémoire ouvrière dans un quartier de Saint-Étienne, 1840-1940, thèse de doctorat d'histoire contemporaine, université Lyon II, 1986, p. 649-653. 
travailleuses des chemins de fer. La construction du genre dans un contexte de labeur connait une singulière application dans les ateliers de réparation ferroviaire, lorsque les ouvrières ont été surtout (mais pas seulement) affectées dans des sections où les tâches étaient perçues comme féminines, comme les selleries ou les bobinages. C'est toute la légende de l'habileté manuelle des " petites mains » qui est ici sollicitée ; or, loin du petit cylindre de fil à coudre, la bobine d'un moteur de locomotive électrique ne nécessite pas véritablement la délicatesse présentée comme une qualité typiquement féminine. Et le travail sur machines à coudre dans les selleries n'évite pas le contact avec la peau tannée, toujours fortement connoté en termes de genre. Cette dimension est sans doute plus importante dans les établissements ferroviaires que dans les usines de guerre des sites métallurgiques, alors que dans ce secteur la très forte croissance des effectifs d'ouvrières pendant la Grande Guerre n'a pas permis que se mette en place une spécialisation à ce point sexuée. Enfin, pour une réelle connaissance du travail dans les sites ferroviaires, l'étude de sa rationalisation est capitale, tentatives menées sur un temps long, celui qui court de la fin du marchandage des tarifs par équipe jusqu'à nos jours, lorsque, là encore, le passage de la traction vapeur à la traction électrique se révèle fondamental.

Une lacune cependant est remarquable. Les aspects syndicaux et ceux des luttes sociales ne sont pas abordés. C'est pourtant un thème où la distinction entre ateliers et dépôts se situe dans une dynamique assez claire, lorsque le flambeau de la lutte des classes passe des ateliers, ces « concentrations prolétariennes exemplaires » telles que les décrivait Annie Kriegel ${ }^{11}$, ces «brigades centrales de la Révolution » dénoncées un parlementaire de droite après la Grande Guerre ${ }^{12}$, aux dépôts, encore à la pointe, seuls d'ailleurs, de la grève du printemps 2001. Le passage du témoin, cette marque et ce transmetteur de l'identité cheminote, s'est fait au cours des années soixante et a changé grandement la nature des grèves dans les chemins de fer ${ }^{13}$. Des travaux de qualités ont été menés sur ce sujet à l'université de Provence (Aix-Marseille I), notamment ceux d'Élö̈se Rougé et de Maud Soulier sur le mouvement social des

11- Annie Kriegel, Aux origines du communisme français, 1914-1920, Paris-La Haye, Mouton, 1964, p. 379.

12- Ibid., p. 449.

13- Christian Chevandier, Cheminots en grève, ou la construction d'une identité (1848-2001), Paris, Maisonneuve et Larose, 2002. 
cheminots marseillais ${ }^{14}$, le numéro 9 d'Industries en Provence, consacré au chemin de fer, comprend un long article de Jean Domenichino et Robert Mencherini consacré au «Syndicalisme cheminot et conflits en Provence $»^{15}$. En dépit de ces recherches, cette absence n'est pas moins un symptôme du désintérêt actuel des historiens pour ces sujets, dédain d'autant plus insolite que les autres chercheurs en sciences sociales, sociologues, psychologues, politistes, ethnologues ne désertent pas ce terrain, conscients qu'ils sont des trésors que l'on peut y découvrir.

Et puisqu'il est question de trésor, ne celons le plaisir que procure cet extraordinaire tableau de Van Gogh accroché au musée Angladon d'Avignon. Ce numéro de la Revue d'histoire des chemins de fer en devient dès lors malaisé à ouvrir, tant ces teintes, ces formes, leurs arrangements nous fascinent. Vincent a aimé peindre le chemin de fer (il faut aller à Moscou admirer au musée Pouchkine le Paysage avec carriole et train au Loin qu'il a peint deux ans plus tard à Auvers-sur-Oise !), mais il le traite rarement comme un sujet central. Même le tableau exposé au musée Rodin, Le Train bleu (ou Vue du viaduc à Arles), peint au mois de mars, nous montre les arbres à la fin de l'hiver bien plus qu'un très discret convoi. Cette toile, Wagons de chemin de fer, il l'a peinte sur place, lors de sa première année en Provence ${ }^{16}$, la plus heureuse. Il s'en fallut de peu que son sujet ne se dérobe. Le dépôt avait été déplacé en Avignon quelques mois plus tôt, et l'année suivante l'activité de l'atelier des voitures était transférée sur d'autres rives du Rhône, à la lumière moins féconde, là où les eaux du fleuve sont gonflées de celles de la Saône. Dès lors, le matériel roulant devint moins commun dans la campagne arlésienne.

Le chemin de fer organise l'espace. Par les réseaux, il suscite ou affermit le dynamisme d'une région. À une autre échelle, il construit la ville, y draîne les hommes, façonne parfois les sociabilités. Le colloque se termine par une visite des Ateliers d'Arles, qu'a préparée pour nous Coralie Grégoire, auteur d'un mémoire de maîtrise sur ce site

14- Éloïse Rougé, Gares et dépôts marseillais, histoire d'un syndicalisme cheminot, 18971937, mémoire de maitrise, 1998 et Le Syndicalisme des cheminots marseillais des années 1960, mémoire de DEA d'histoire contemporaine, université de Provence, 1999; Maud Soulier, Le Mouvement social aux Ateliers de Marseille-Prado de 1875 à 1937, mémoire de maîtrise d'histoire contemporaine, université de Provence, 1999.

15- Jean Domenichino et Robert Mencherini, «Syndicalisme cheminot et conflits en Provence », Industries en Provence, $\mathrm{n}^{\circ} 9$ (avril 2002), p. 15-21.

16- Un dessin représentant une scène fort proche, vue de l'autre côté du train, le Paysage aux poteaux télégraphiques est conservé à la Fondation Vincent Van Gogh d'Amsterdam. Ce dessin et la toile datent tous deux de l'été 1888. 
ferroviaire ${ }^{17}$. Loin d'une démarche touristique, cette " descente » sur place est bien ce que les historiens doivent emprunter aux géographes. Pour quelle raison nous priverions-nous de cette source de la connaissance de l'espace humain qu'est la sortie sur le terrain ? Comme l'ethnologue, l'historien doit pouvoir se mettre en situation. L'appréhension spatiale lui est nécessaire parce que les hommes sont situés dans un (des) espace(s), et que, ainsi que le soulignait Marc Bloch, « ce sont des hommes que l'histoire veut saisir ». Le jeu d'échelle est devenu un outil de l'historien. Pour l'historien des chemins de fer, il l'est de longue date, tant il est vrai que l'organisation par réseau le rend indispensable. Il a été renouvelé par les travaux menés récemment dans la région Provence-Alpes-Côte d'Azur, dont la publication l'an dernier de Cheminots en Provence ${ }^{18}$, largement diffusé, a été le couronnement. Pour l'étude des ateliers et dépôts du Matériel ferroviaire, les différentes recherches incitent à plaider pour une descente sur le terrain. Tout ce qui nous est écrit dans ce recueil, la réalité des établissements, le travail, la production, la perception même que travailleurs et habitants des environs en avaient, tout cela est situé dans un espace. En transposant le colloque dans un espace, celui d'un établissement, c'est aussi pour le renforcement d'une ouverture pluridisciplinaire, largement entamée comme le prouvent les spécialités des auteurs de communications, que nous prêchons.

17- Coralie Grégoire, Les Ateliers d'Arles de 1848 à 1984, 136 ans d'activité industrielle au service de la cause ferroviaire, mémoire de maitrise d'histoire contemporaine, université de Provence, 2001.

18- Robert Mencherini et Jean Domenichino avec David Lamoureux, Cheminots en Provence. Des voix de la mémoire aux voies de l'avenir (1830-2001), Paris, Éditions La Vie du Rail, 2001. 


\section{Oullins, l'usine des cheminots}

Édifiés au milieu des années 1840 sur la rive droite du Rhône, un peu en aval de la confluence avec la Saône, les Ateliers ferroviaires d'Oullins se sont consacrés dans un premier temps à la construction mécanique, de ponts roulants, de bateaux, de locomotives, avant de se spécialiser une décennie plus tard dans la construction de matériel ferroviaire, puis à des activités exclusives de réparation à partir de 1891. Près du principal atelier, celui d'Oullins-Machines où étaient réparées les locomotives, ouvrait en 1889 une seconde usine pour la réparation du matériel roulant, celle d'Oullins-Voitures, dont l'activité cessa un siècle plus tard. Exploité par la Compagnie du PLM à partir de 1861, le site appartint à la SNCF dès la nationalisation. Le principal bouleversement de l'histoire de cette immense usine fut, en 1948, la reconversion d'Oullins-Machines à la réparation des locomotives électriques. L'ensemble employait plus de 4500 personnes à la fin de la Deuxième Guerre mondiale ; au début de xxi siècle, ils sont un peu plus d'un millier de cheminots à travailler sur le site d'Oullins-Machines.

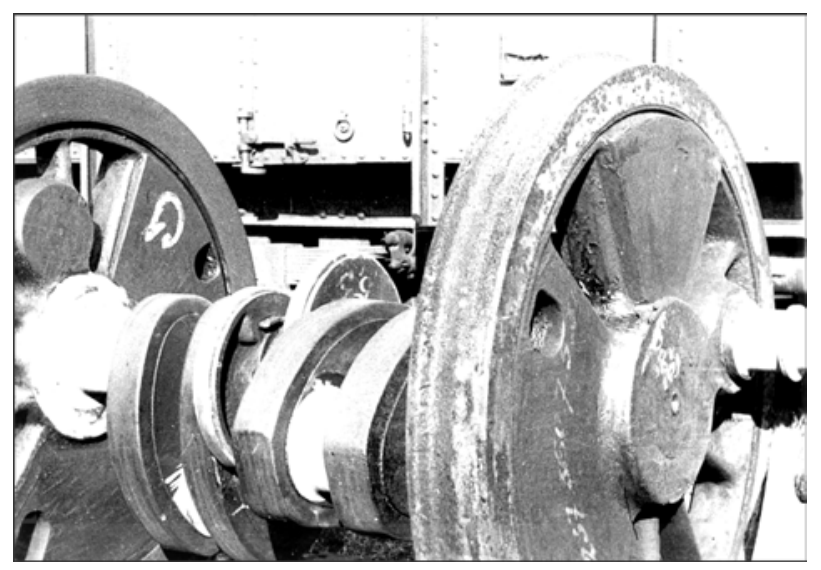

Figure 1. À l'atelier des roues d'Oullins-Machines. Les contraintes de la sécurité et la nécessité d'obtenir des alliages très fiables ont amené la Compagnie du PLMà conserver l'activité de fabrication des roues au sein de ses ateliers pourtant consacrés exclusivement aux activités de réparation. Cl. Ch. Chevandier, 1986. 


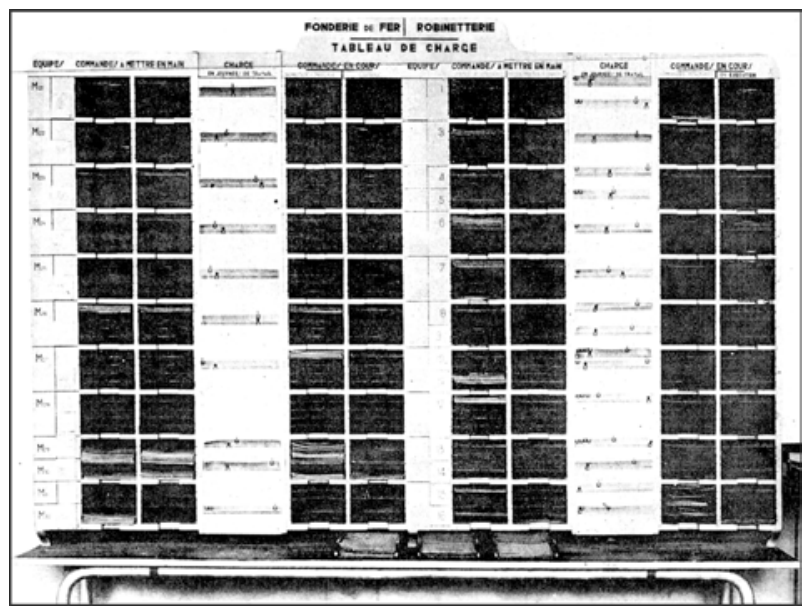

Figure 2. Un tableau de planification aux fonderies d'Oullins-Machines dans I'entre-deux-guerres. Après la Première Guerre mondiale, les ateliers ferroviaires ont joué un rôle précurseur dans l'organisation du travail industriel en France. Cl. PLM, coll. Ch. Chevandier.

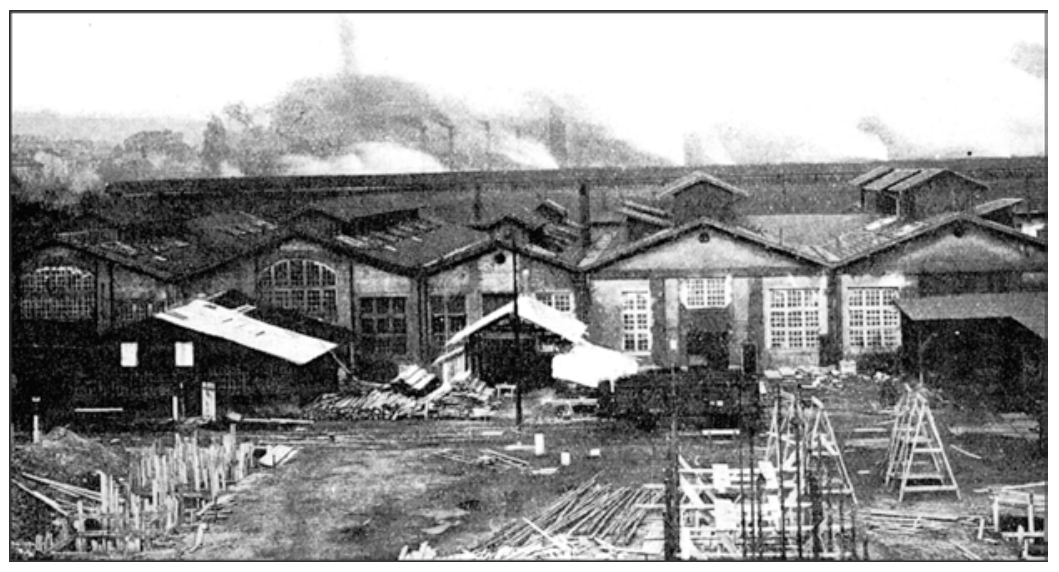

Figure 3. L'ancienne fonderie de fonte et le début de la construction du nouveau bâtiment aux ateliers d'Oullins-Machines à la fin des années 1920. Les sites ferroviaires, souvent très anciens et dont une partie des bâtiments était en bois, ont été rénovés sans interruption de l'activité. Cl. PLM, coll. Ch. Chevandier. 


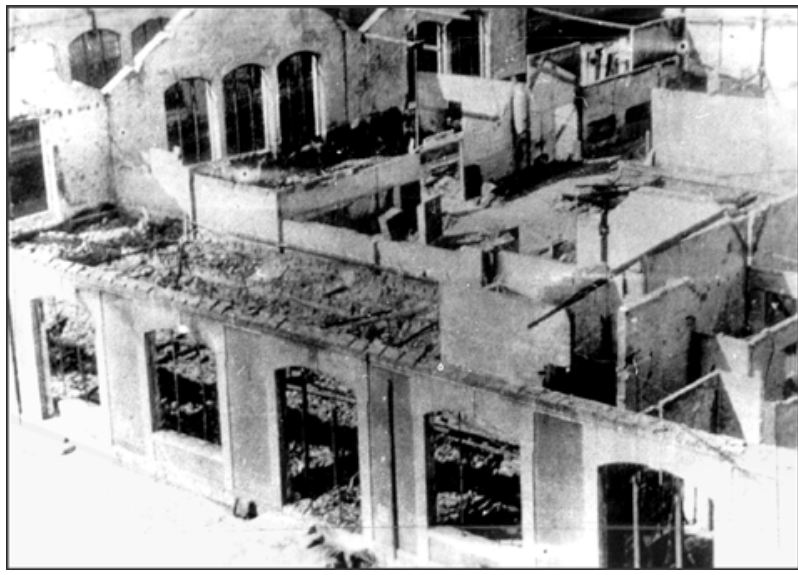

Figure 4. Les bureaux d'Oullins-Machines incendiés à la Libération par les troupes allemandes en retraite. Les installations ferroviaires ont subi de considérables dégâts au cours de la Seconde Guerre mondiale. Ce fut souvent l'occasion d'une modernisation du patrimoine immobilier et d'une adaptation du bâti. Cl. d.r., coll. Ch. Chevandier.

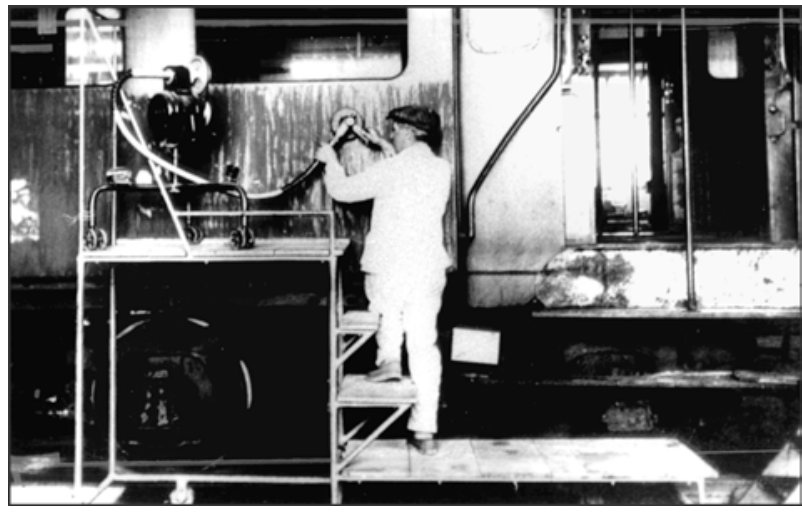

Figure 5. Un ouvrier en train de décaper une voiture à Oullins-Voitures, milieu des années 1930. Une multitude de corps de métiers exerçait dans les grands ateliers du Matériel. Cl. PLM, coll. Ch. Chevandier. 


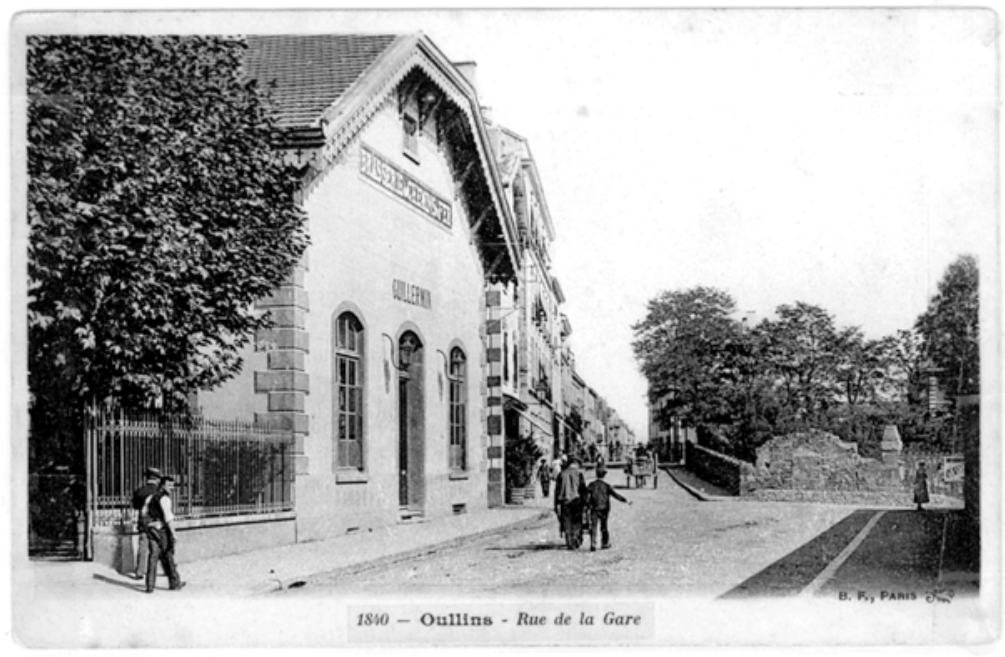

Figure 6. La Brasserie des Chemins de fer, rue de la Gare à Oullins. Autour des grands sites ferroviaires l'espace urbain était fortement marqué, y compris par la toponymie. Cl. B.F., coll. Ch. Chevandier.

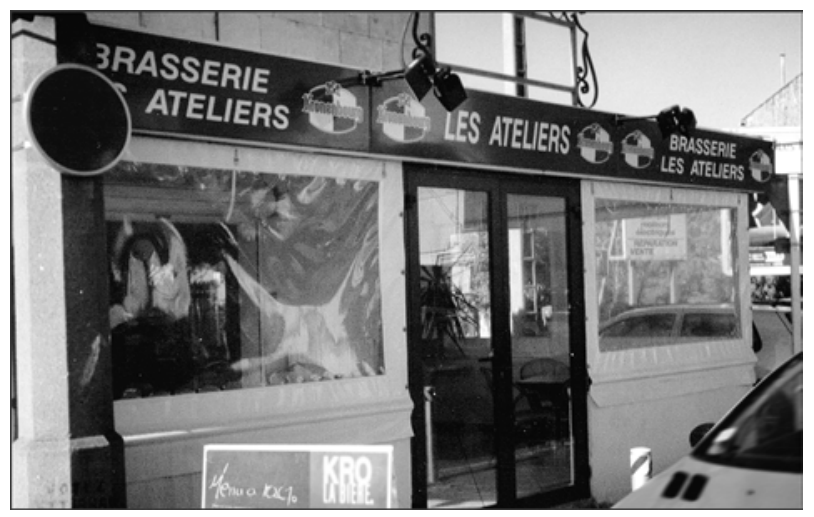

Figure 7. La Brasserie "Les Ateliers" en Arles, avril 2002. Bien après la fin de l'activité, la mémoire en est maintenue par de nombreux biais. Cl. M.-N. Polino/AHICF. 